\title{
Orbit Reconstruction with 3D Printed PEKK Implant
}

\author{
Rachel Armstrong, $B A^{1}$ and Marco Ellis, $M D^{2 *}$ \\ ${ }^{1}$ Feinberg School of Medicine, Northwestern University, Chicago, IL, USA \\ ${ }^{2}$ Division of Plastic Surgery, Feinberg School of Medicine, Northwestern University, Chicago, IL, USA
}

\begin{abstract}
Introduction: Alloplastic materials are used in facial bony reconstruction, but the ideal material for such procedures has yet to be elucidated. 3D printed polymers are a promising new material for use in bony reconstructive procedures. This article documents the novel usage of polyetherketoneketone (PEKK) in an orbital floor reconstruction.
\end{abstract}

Report of case: We report the case of a patient who experienced a traumatic left-sided orbital floor fracture and received a porous polyethylene implant. This inadequately restored the orbital floor, and the patient underwent a revision surgery of this defect with PEKK.

Discussion and conclusion: This is the first case to demonstrate PEKK use in facial bony reconstruction. The patient's condition improved remarkably after revision surgery with this material, which is encouraging for the future use of 3D printed polymers in reconstruction procedures.

\section{Introduction}

A variety of materials can be used in bony reconstruction of the orbit $[1,2]$. Autologous grafts are widely utilized, as are alloplastic materials including hydroxyapatite, porous polyethylene, and titanium [1,3]. While these materials are widely accepted, each carry a unique risk-benefit profile. Autologous bone grafts are highly biocompatible and have reduced risk of infection, but have disadvantages including donor site morbidity, difficulty with contouring and unpredictable resorption in the long term $[2,4]$. Titanium mesh more accurately restores orbital defects when compared to bone [5], but its usage is associated with orbital adherence syndrome $[6,7]$. Polydioxanone foil results in better post-operative outcomes than titanium implants, yet is most efficacious in defects between $250-300 \mathrm{~mm}^{2}$ [8]. Porous polyethylene is biocompatible and can be easily shaped and fixated $[9,10]$. However, porous polyethylene has also been documented to result in unsatisfactory appearances, foreign body granuloma formation, and infection $[4,11,12]$. Hydroxyapatite similarly carries a high infection rate likely due to loss of structural integrity over time [13]. This was just a brief review of some of the implant materials currently available. In sum, the search for the ideal material is still under way.

Several studies have detailed the safety and effectiveness of 3D printed polymers in cranioplasty [14,15], spinal implants [16], and mandibular defect repair [17]. Polymers, such as polyetheretherketone (PEEK) and polyetherketoneketone (PEKK), are radiolucent, biomechanically stable, and can be fabricated to fit a unique defect, resulting in high-quality aesthetic outcomes [16,18]. A 2009 paper by Kim, et al. detailed the use of patient specific PEEK implants in four different maxillofacial reconstruction cases. PEEK implants improved facial contours and restored bony structures in the orbitomaxillary, superior orbital cranial, and anterior frontal sinus regions [19]. While PEEK has a longer history of use, PEKK shares similar biomechanical characteristics and has been shown to have favorable antibacterial properties [20]. In addition, this material has also been shown to support a high degree of bone apposition [21].

The literature documenting PEEK use in facial bony defects, mandibular repair, and spinal implants provides promise for PEKK's use in similar cases. In this paper, we document the usage of a patient specific PEKK implant in a revision surgery addressing an orbital floor fracture due to left-sided face trauma.

\section{Case Presentation}

The patient was a 50-year-old man who presented with

*Corresponding author: Marco Ellis, MD, Assistant Professor of Surgery, Division of Plastic Surgery, Feinberg School of Medicine, Northwestern University, 675 N Saint Clair St, Suite 19250, Chicago, IL 60611, USA

Accepted: October 16, 2019

Published online: October 18, 2019

Citation: Armstrong R, Ellis M (2019) Orbit Reconstruction with 3D Printed PEKK Implant. J Head Neck Surg 2(1):38-41 
orbital dystopia and discomfort related to a malpositioned orbital floor implant. In summary, the patient sustained an orbital floor fracture after a motor vehicle collision with a deer two months prior to presentation. His initial treatment included fracture reconstruction with Caldwell-Luc maxillary antrostomy and balloon approximation of the floor fracture at another institution two weeks after the accident. A second treating physician diagnosed the patient with ongoing significant floor displacement with prolapse of orbital contents into maxillary sinus six weeks after original repair. The decision was made to place a porous polyethylene implant to restore inadequately reduced orbital volume.

The patient presented to us one month later for urgent evaluation after developing pain and fullness in his lower eyelid. Cross-sectional imaging demonstrated incomplete

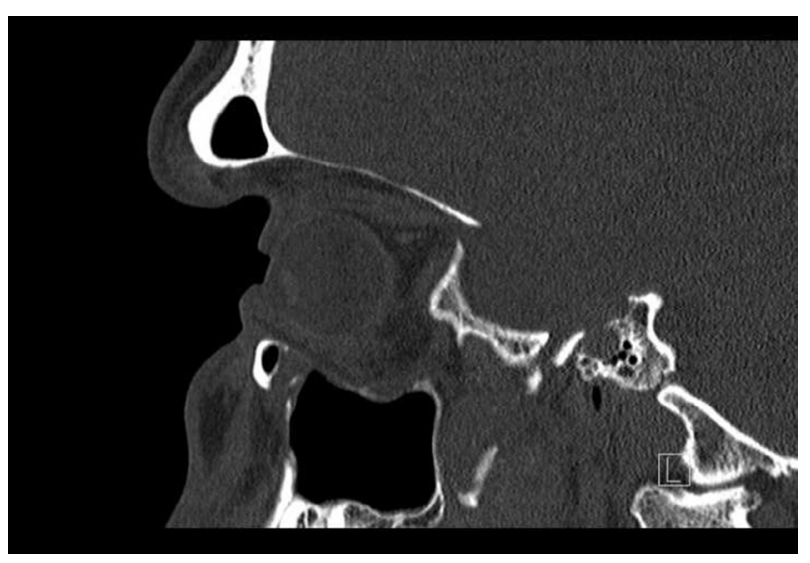

Figure 1: Preoperative CT Face, midsagittal view of left orbit.

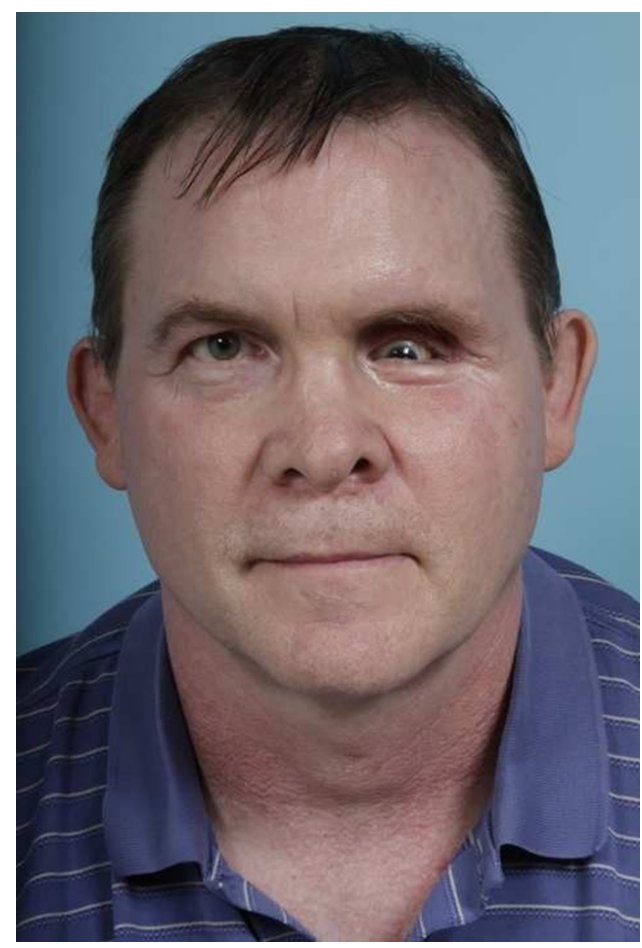

Figure 2: Preoperative appearance with severe orbital dystopia and enophthalmos. reconstruction of the orbit with evidence of a radiolucent implant CT showed inadequate reduction of the orbital floor and medial orbital wall (Figure 1). The implant was cantilevered on the orbital rim with its anterior portion protruding into the orbicularis. The posterior aspect failed to reach the keystone area with orbital contents still herniating into the maxillary sinus. On exam, the patient had severe vertical dystopia with his visual axis coincident with the inferior orbital rim (Figure 2). He had diplopia at rest and with gaze in all directions in addition to mild left V2 numbness and paresthesia. After counseling and discussion, the patient agreed to implant removal and revision with a 3D-printed implant. After virtual surgical planning (Figure 3), the patient underwent repeat surgery through a transconjunctival, retroseptal approach.

Reconstruction was performed with a patient-specific 3-mm thick PEKK implant designed to recreate the orbital floor and medial orbital wall. In addition, the implant augmented the inferior orbital rim where it was previously

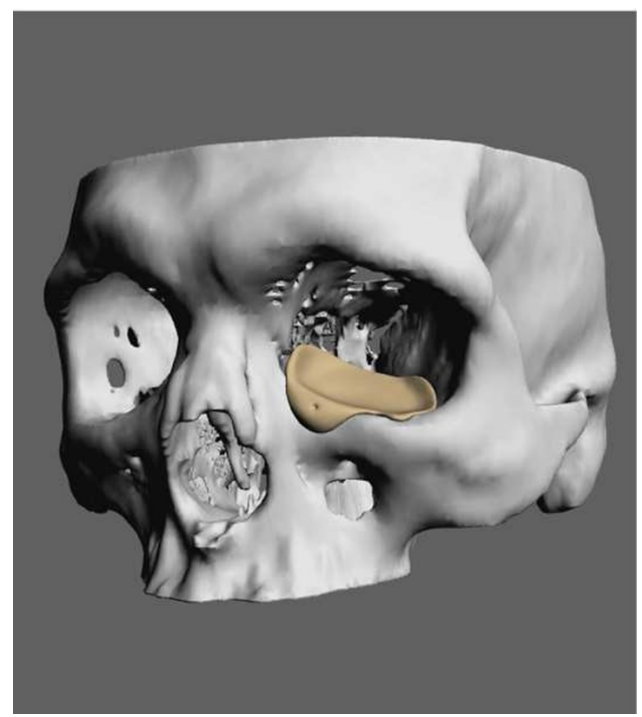

Figure 3: Virtual 3D image demonstrating planned implant reconstruction of orbital floor, medial orbital wall and inferior orbital rim.

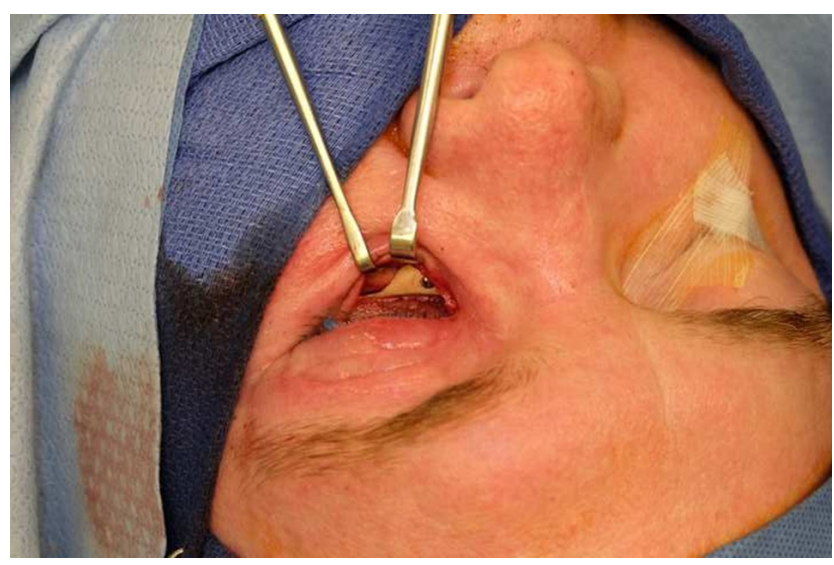

Figure 4: Intraoperative view of PEKK implant fixated along inferomedial orbital rim with transconjunctival access. 


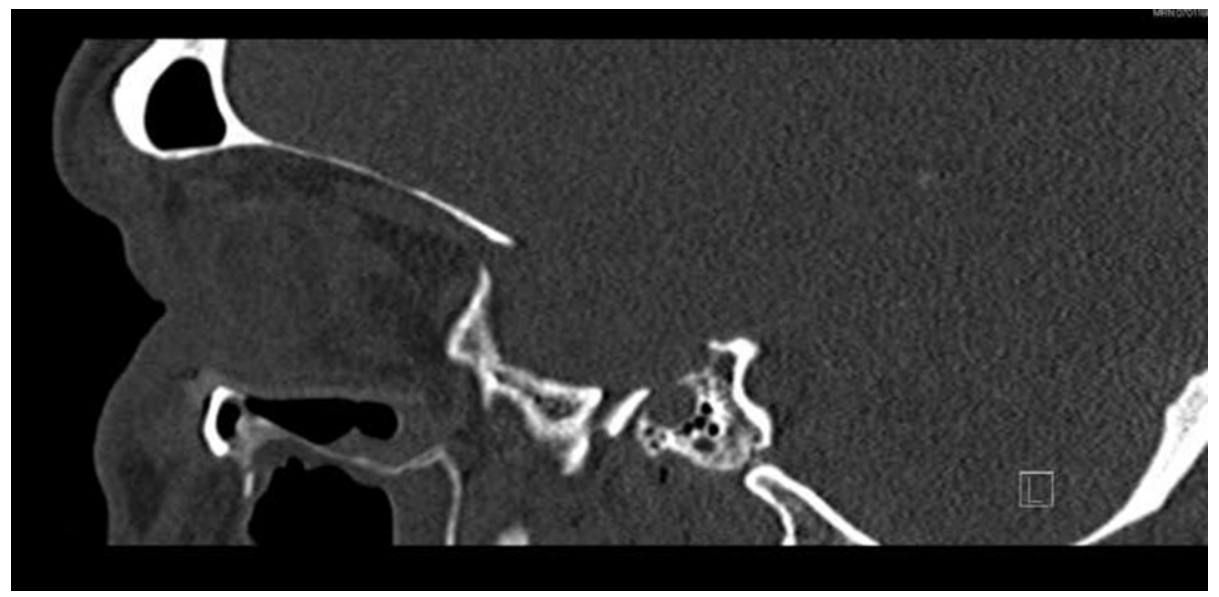

Figure 5: Postoperative CT Face, midsagittal view of left orbit.

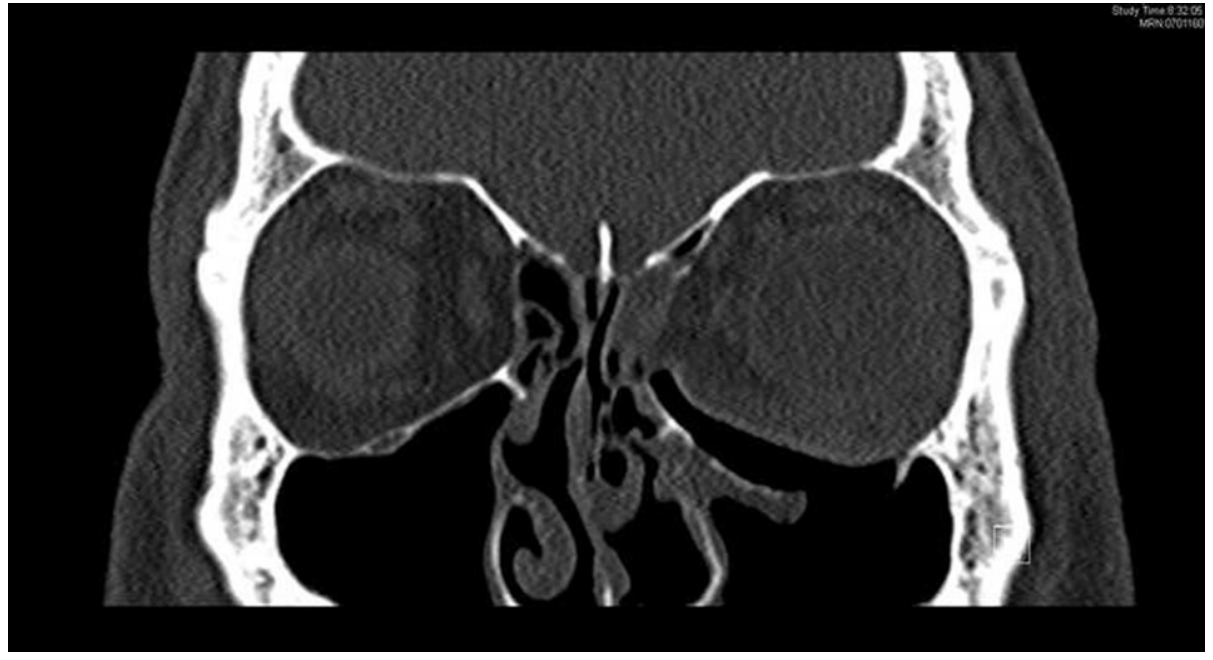

Figure 6: Postoperative CT Face, coronal view.

fractured (Figure 4). Fixation was performed with a single 4-mm titanium screw. At follow up one month later (Figure 5 and Figure 6), the patient continued to have pseudo-enophthalmos and hypoglobus despite our bony reconstruction. The patient had developed severe periorbital fat atrophy. Six months later he underwent revision surgery with stacked porous polyethylene sheets to improve the soft tissue-bony volume relationship. He also required ectropion repair with midface flap elevation, suspension and acellular dermal matrix graft for posterior lamella interposition. His postoperative course for both procedures were uncomplicated with respect to major medical or surgical complications. At 16-month follow-up, the patient presented with stabilization of the orbital floor and with improved but persistent 2-mm hypoglobus and enophthalmos. His vision is drastically improved with some diplopia at extreme gaze. He does not require prisms to activities of daily living nor has he required any strabismus procedures.

\section{Discussion}

Although there is data to support the use of polymer implants in the body globally, this is the first report to document the use of PEKK in the face and around the sinus mucosa. In this case, PEKK was used in the revision of an orbital floor and medial wall blow out fracture that was previously inadequately addressed by porous polyethylene. While the patient continues to have some asymmetry likely due to the severity of his injury and multiple operations, the patient specific PEKK implant improved his vision and orbit position.

PEEK implants have been used in the face, sinus mucosa, and orbital floor. PEKK has similar biocompatibility to PEEK but has some unique advantages. It has been shown to possess antibacterial properties and promotes bone apposition $[20,21]$. PEKK can be printed in simple and elaborate shapes with varying thicknesses. It can also be smoothened or dyed based on clinical applications. Our center continues to research PEKK's role in bony reconstruction and augmentation of the head and neck. Further research is needed to make concrete conclusions about PEKK's efficacy and to determine long term outcomes. Long term data with a similar variant to PEKK suggest a safe profile in a variety of surgical contexts $[19,22-24]$. Based off of this case report, PEKK holds much promise. 


\section{Conflicts of Interest}

None declared.

\section{References}

1. Avashia YJ, Sastry A, Fan KL, et al. (2012) Materials used for reconstruction after orbital floor fracture. J Craniofac Surg 23 : 1991-1997.

2. Dubois L, Steenan SA, Gooris PJJ, et al. (2016) Controversies in orbital reconstruction-III. Biomaterials for orbital reconstruction: A review with clinical recommendations. Int J Oral Maxillofac Surg 45: 41-50.

3. Friedman CD, Costantino PD, Snyderman CH, et al. (2000) Reconstruction of the frontal sinus and frontofacial skeleton with hydroxyapatite cement. Arch Facial Plast Surg 2: 124-129.

4. Mok D, Lessard L, Cordoba C, et al. (2004) A review of materials currently used in orbital floor reconstruction. Can J Plast Surg 12: $134-140$.

5. Ellis E, Tan $Y$ (2003) Assessment of internal orbital reconstructions for pure blowout fractures: Cranial bone grafts versus titanium mesh. J Oral Maxillofac Surg 61: 442-453.

6. Lee HB, Nunery WR (2009) Orbital adherence syndrome secondary to titanium implant material. Ophthalmic Plast Reconstr Surg 25: 33-36.

7. Kersey TL, Ng SG, Rosser P, et al. (2013) Orbital adherence with titanium mesh floor implants: A review of 10 cases. Orbit 32 8-11.

8. Holtmann H, Eren H, Sander K, et al. (2016) Orbital floor fractures-short- and intermediate term complications depending on treatment procedures. Head Face Med 12: 1.

9. Marano R, Tincani AJ (2016) Is there an ideal implant for orbital reconstructions? Prospective 64-case study. J Craniomaxillofac Surg 44: 1682-1688.

10. Rubin PA, Bilyk JR, Shore JW (1994) Orbital reconstruction using porous polyethylene sheets. Ophthalmology 101: 1697-1708.

11. Ridwan-Pramana A, Wolff J, Raziei A, et al. (2015) Porous polyethylene implants in facial reconstruction: Outcome and complications. J Craniomaxillofac Surg 43: 1330-1334.

12. Timoney PJ, Clark JD, Frederick PA, et al. (2016) Foreign body granuloma following orbital reconstruction with porous polyethylene. Ophthal Plast Reconstr Surg 32: e137-e138.

13. Mathur KK, Tatum SA, Kellman RM (2003) Carbonated apatite and hydroxyapatite in craniofacial reconstruction. Arch Facial Plast Surg 5: 379-383.

14. Ng ZY, Nawaz I (2014) Computer-designed PEEK implants: A peek into the future of cranioplasty? J Craniofac Surg 25: e55-e58.

15. Scolozzi P, Martinez A, Jaques B (2007) Complex orbito-fronto-temporal reconstruction using computer-designed PEEK implant. J Craniofacial Surg 18: 224-228.

16. Kurtz SM, Devine JN (2007) PEEK biomaterials in trauma, orthopedic, and spinal implants. Biomaterials 28: 4845-4869.

17. Roskies MG, Fang D, Abdallah MN, et al. (2017) Three-dimensionally printed polyetherketoneketone scaffolds with mesenchymal stem cells for the reconstruction of critical-sized mandibular defects. Laryngoscope 127: E392-E398.

18. Rosenthal G, Ng I, Moscovici S, et al. (2014) Polyetheretherketone implants for the repair of large cranial defects: A 3-center experience. Neurosurgery 75: 523-529.

19. Kim MM, Boahene KD, Byrne PJ (2009) Use of customized polyetheretherketone (PEEK) implants in the reconstruction of complex maxillofacial defects. Arch Facial Plast Surg 11: 53-57.

20. Wang M, Bhardwaj G, Webster TJ (2017) Antibacterial properties of PEKK for orthopedic applications. Int J Nanomedicine 12: 6471-6476.

21. Hacking SA, De la Vega R, Bubar C (2014) Tissue response to 3D printed PEKK polymer implants. 60th Annual ORS Meeting, New Orleans LA, USA.

22. Jalbert F, Boetto $S$, Nadon F, et al. (2014) One-step primary reconstruction for complex craniofacial resection with PEEK custom- made implants. J Craniomaxillofac Surg 42: 141-148.

23. Punchak M, Chung LK, Lagman C, et al. (2017) Outcomes following polyetheretherketone (PEEK) cranioplasty: Systemic review and meta-analysis. J Clin Neurosci 41: 30-35.

24. Chen Y, Wang X, Lu X, et al. (2013) Comparison of titanium and polyetheretherketone (PEEK) cages in the surgical treatment of multilevel cervical spondylotic myelopathy: A prospective, randomized, control study with over 7-year follow-up. Eur Spine J 22: 1539-1546.

DOI: $10.36959 / 605 / 535$

Copyright: (C) 2019 Armstrong R, et al. This is an open-access article distributed under the terms of the Creative Commons Attribution License, which permits unrestricted use, distribution, and reproduction in any medium, provided the original author and source are credited. 